\title{
Design Maps of TRPM8 as a Thermometer for Cold Detection
}

\author{
Guangyu Wang* \\ Department of Physiology and Membrane Biology, University of California School of Medicine, \\ Davis, CA, USA \\ Department of Drug Research and Development, Institute of Biophysical Medico-chemistry, \\ Reno, NV, USA \\ * Author to whom correspondence should be addressed; E-mail: gary.wang10@ gmail.com
}

\begin{abstract}
The menthol sensor TRPM8 can be activated by cold and thus serves as a thermometer in a primary afferent sensory neuron for noxious cold detection. However, the underlying design principle is unknown. Here, a hairpin topological structural model and graph theory were prepared to test a role of the cold-dependent hairpin formation in the cold-evoked gating pathway of TRPM8. The results showed that the formation of a large lipid-dependent hairpin initiates a low temperature threshold in favor of TRPM8 activation. Furthermore, two smaller hairpins, which enhance the coupled interactions of the voltage-sensor-like domain with both the pore domain and the TRP domain, can stabilize the cold efficacy and work as a fuse to prevent cold denaturation. The coldinduced hairpin rearrangements along the gating pathway may be necessary for the high cold sensitivity. This hairpin model may provide a structural basis for activation of the thermo-gated TRP channels at low temperature. (150 words)
\end{abstract}

Keywords: allosteric gating | graph theory | hairpin formation | ion channel | noncovalent interaction I temperature threshold 


\section{INTRODUCTION}

Transient receptor potential (TRP) melastatin-8 (TRPM8) is a $\mathrm{Ca}^{2+}$-permeable nonselective cation channel, serving as a polymodal sensor in response to both physical and chemical stimuli. The physical stimuli include voltage and cold while the chemical stimuli cover phosphatidylinositol-4, 5-biphosphate $\left(\mathrm{PIP}_{2}\right)$ and cooling agents such as menthol and its analogs WS-12 and icilin. ${ }^{[1-9]}$ As a noxious cold sensor in a mammalian peripheral afferent neuron of the dorsal root and the trigeminal ganglia, TRPM8 has a high temperature sensitivity (Q10 of 40 at pH 6.7) but an activation threshold $20-28^{\circ} \mathrm{C}$ below a normal human body temperature $37^{\circ} \mathrm{C} .{ }^{[10-15]}$ Since the TRPM8 channel can be activated by both cold and menthol stimuli in a reversible manner, noncovalent interactions along the gating pathway in TRPM8 may be determinant. However, much less is known about whether both cooling stimuli share the same pathway to gate TRPM8, and what governs those non-covalent interactions during TRPM8 gating. When heat can decrease the menthol-evoked activity of TRPM8 or menthol can sensitize to cold and thus increase the temperature threshold and the saturation temperature of rat TRPM8 (rTRPM8) from $22-26^{\circ} \mathrm{C}$ and $8.2^{\circ} \mathrm{C}$ to $30-32^{\circ} \mathrm{C}$ and $15.6^{\circ} \mathrm{C}$, respectively, the same gating pathway seems to be shared. ${ }^{[1-2,10,16]}$ However, some menthol-sensitive residues such as Y745 and Y1005 in mouse TRPM8 (mTRPM8) are insensitive to cold, challenging the same gating pathway for both stimuli. ${ }^{[17]}$

$\mathrm{Ca}^{2+}$-bound TRPM8 from Parus major (pmTRPM8) has produced the highest resolution for the full-length cryo-electron microscopy (cryo-EM) structural model. ${ }^{[18]}$ This homotetrameric TRPM8 channel has the transmembrane channel layer to interact with a large two-layered cytosolic ring formed by a large N-terminal melastatin homology regions (MHR1-MHR4) and a C-terminal coiled-coil. The transmembrane channel region has a voltage-sensor-like domain (VSLD) with S1-S4 and a pore domain with S5-S6 and the intervening pore helix and a much longer pore loop to form a domain-swapped tetramer, with the VSLD from one subunit interacting with the pore domain of the adjacent one. The channel pore includes the negatively charged selectivity filter at the outer leaflet of the membrane as the upper gate and the S6 bundle crossing within the inner leaflet as the lower gate. The canonical S4-S5 linker and the S6-extended TRP domain are also present for critical channel gating with enough resolution. ${ }^{[18]}$ Unlike TRPV1, the pre-S1 domain of TRPM8 includes the cytosolic pre-S1 helix and a helix-turn-helix (HTH) followed by an interfacial helix that connects to $S 1 .{ }^{[18-19]}$ 
TRPM8 has many aromatic and aliphatic residues between the VSLD and the TRP domain to form a large hydrophobic pocket for the binding of the cooling agents such as menthol and WS12 and icilin. ${ }^{[21-23]}$ It has been reported that menthol-sensitive R842 in this pocket affects colddependent activation of human TRPM8 (hTRPM8). ${ }^{[7]}$ On the other hand, capsaicin or vanillyl butyl ether (VBE), a capsaicin analog, can activate human TRPV1 but inhibit cold-evoked hTRPM8 currents and menthol-sensitive Y745 in this pocket is involved. ${ }^{[24]}$ Therefore, both menthol and cold may employ the same hydrophobic pocket but with distinct sensitive residues to illicit the cooling signal. Since the temperature-dependent gating of the TRPM8 channel is similar to a melting curve of a DNA hairpin thermal sensor, and the increased hairpin loop size can lower the melting temperature, ${ }^{[10,25-26]}$ it is attractive to use non-covalent interactions in the available cryo-electron microscopy (cryo-EM) structures of frozen TRPM8, together with graph theory, to examine if the cold-dependent hairpin formation along the gating pathway facilitates TRPM8 opening, and which residue is sensitive to cold (Figure 1). On the other hand, intracellular acidic pH6 can prevent cold-evoked activation of TRPM8. ${ }^{[10]}$ Therefore, a putative swapping transition metal bridge between the VSLD and the pore domain, which may be disrupted by the acidic pH6, is proposed to stabilize the cold efficacy. The resultant smaller hairpins may protect the TRPM8 channel from cold denaturation. The role of $\mathrm{Ca}^{2+}$ and $\mathrm{PIP}_{2}$ regulatory sites in and beside this menthol pocket in mediating different gating states were also explored and discussed, respectively. [18, 21] The design maps created in this study may provide further insight into the molecular structural basis for thermosensitive TRP channels as a biological thermometer.

\section{RESULTS}

\subsection{The Non-covalent Interactions along the Gating Pathway Form the Primary Hairpin

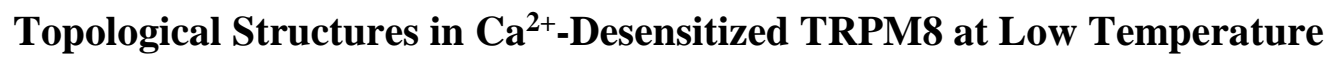

The cryo-EM structures of $\mathrm{Ca}^{2+}$-desensitized pmTRPM8 in a frozen state are now available with enough resolution to examine how non-covalent interactions along the gating pathway form primary hairpin topological structures to prime cold activation. ${ }^{[18]}$ First, several pairwise residues can provide the salt bridges to form hairpins. In VSLD, salting pairs have R832 on S4 and D793 on S2, R807 on S3 and E759 on S2, and K753 in the S1-S2 loop and E812 in the S3-S4 loop. In the pore domain, salt-bridging pairs cover R875 on S5 and E896 on the pore helix or D910 on the pore loop, E932 on the pore loop and R880 between S5 and the pore helix, D908 and R918, D917 and K927, R940 and E932 or D934 in the pore loop (Figure 2). 
Second, $\pi-\pi$ interactions between aromatic side rings along the gating pathway also generate hairpins (Figure 2). In the VSLD and its interfaces with the pre-S1 domain and the TRP domain, there are three groups with edge-face or face-face $\pi-\pi$ interactions between different helix segments. The first includes W673 on pre-S1, F726 and F729 on S1, and F837 on S4; the second has F739 and F743 on S1, Y763 on S2, F800 on S3, F822 and F826 and F829 on S4; the third contains W777 on S2, Y784 and W789 in the S2-S3 linker, and H835 on S4. In contrast, the pore domain has F871 on S5 and Y953 on S6 or Y898 and F902 on the pore helix as face-face or edgeface $\pi-\pi$ interactions (Figure 2 ).

Third, H-bonds between different segments also form hairpins. These pairwise H-bonding residues are R988 in the TRP domain and Q666 on pre-S1, Y736 on S1 and R832 on S4, D750 and T756 in the S1-S2 loop, N790 on S3 and D787 or T794 on S3, R852 in the S4-S5 linker and N980 in the TRP domain, D856 on S5 and T972 on S6, Q876 on S5 and S892 or E896 on the pore helix, and T920 and E925 in the pore loop (Figure 2).

Finally, the famous $\mathrm{Ca}^{2+}$ site in the menthol pocket also links E773 and Q776 on S2, Y784 in the S2-S3 linker, and D793 and N790 on S3 together (Figure 2). Totally, there are four hairpin groups with the smallest loop sizes to govern non-covalent interactions along the gating pathway: the first group with 0 to 16-residue loops in the outer pore; the second group with 3 to 21 -residue loops between S5 and S6; the third group with 2 to 29-residue loops from the TRP domain to the pre-S domain and the VSLD and the S4-S5 linker; the fourth group with 0 to 21-residue loops in the VSLD (Figure 2). Those two intra-domain interactions such as the F837-F729 $\pi-\pi$ interaction in VSLD and the R852-N980 H-bond in the pore domain may favor the formation of a large hairpin with a 29-residue loop in frozen TRPM8. However, they are unrelated to the relative movement of the S4-S5 linker against the TRP domain for activation of TRPM8 at low temperature.

\subsection{The Inter-domain Interactions Are Required for a Large Hairpin to Facilitate Cold Activation of TRPM8 at a Low Temperature Threshold}

Intracellular $\mathrm{Ca}^{2+}$ is not needed for cold- or menthol-dependent TRPM8 activation. ${ }^{[1,4]}$ However, $\mathrm{Ca}^{2+}$ binding with a high affinity was proposed to disrupt a putative $\mathrm{H}$-bond between R998 in the TRP domain and Q776 on S2 and thus to desensitize the menthol-evoked TRPM8 activity. ${ }^{[18]}$ Therefore, it is necessary to examine if the R998-Q776 H-bond is present in $\mathrm{Ca}^{2+}$-free TRPM8 with a menthol analog WS-12 bound. 
In the absence of $\mathrm{Ca}^{2+}$ but presence of WS-12, the cryo-EM structure is different in several ways when compared to the $\mathrm{Ca}^{2+}$-desensitized pmTRPM8 (Figure 3). First, the $\mathrm{Ca}^{2+}$ bridge between S2 and S3 is disrupted. Instead, the equivalent side chain H-bonds between Y784 and E773/T794 are emerging. Second, the equivalent aromatic $\pi-\pi$ interactions between F800 and Y763 or F822, Y826 and F743, F837 and F729, and F871 and Y953 are also disconnected. In the meantime, the equivalent edge-face $\pi-\pi$ interaction between F729 on S1 and Y995 in the TRP domain is present. Third, while the equivalent D793-R832 salt bridge is replaced with the D793R832 H-bond, and the equivalent T756-D750 and R832-Y736 and R852-N980 and D856-T972 H-bonds are broken, the equivalent side chain H-bond between R998 in the TRP domain and Q776 on S2 is indeed generated. Simultaneously, Q851 on S5 H-bonds with Q987 in the TRP domain. In this way, an equivalent larger hairpin is shaped with a about 29-residue loop from R998 to Q776, W777, Y784, T794, W789/D793, H835/R832, Q851, and Q987, in favor of a low temperature threshold to initiate cold activation of TRPM8 (Figure 3).

\subsection{The Putative R998-Q776 H-bond between the TRP Domain and the VSLD IS Needed for Cold Activation of TRPM8}

Capsaicin or its analog VBE has been reported to activate hTRPV1 but to inhibit coldevoked hTRPM8 currents by targeting menthol-sensitive Y745 (Y736 in pmTRPM8) in the WS12 or menthol or icilin pocket of hTRPM8. ${ }^{[21,23-24]}$ The in silicon molecular docking demonstrates that in the $\mathrm{Ca}^{2+}$-desensitized state, the vanillyl methoxy group of capsaicin or VBE can H-bond with the hydroxyl group of Y736 of pmTRPM8 while their aromatic rings can form an edge-face $\pi-\pi$ interaction with the aromatic ring of Y736. In the meanwhile, the amide oxygen of capsaicin or the ether oxygen of VBE can H-bond with the side chains of E773 on S2 or R998 in the TRP domain. In this manner, the lipidic tail of capsaicin or VBE can point toward the crevice between R998 and Q776 to stop the formation of the putative R998-Q776 H-bond (Figure 4A). Therefore, the putative stimulatory R998-Q776 H-bond may be indispensable regarding cold sensing of TRPM8. In agreement with this notion, R998 cannot H-bond with Q776 in the $\mathrm{Ca}^{2+}$-desensitized state (Figure 4A).

\subsection{PIP 2 is Necessary to Bridge the TRP Domain with the Pre-S1 Domain for Cold-Evoked Activation of TRPM8}

$\mathrm{PIP}_{2}$ is required for TRPM8 activation because depletion of $\mathrm{PIP}_{2}$ by poly-lysine can desensitize the channel. ${ }^{[5-7,9,27]}$ In contrast, PIP 2 alone can activate vertebrate TRPM8, possibly 
by interacting with K995 (K985 in pmTRPM8) and R998 (R988 in pmTRPM8) in the TRP domain. [5, 21] Although the stimulatory R998-Q776 H-bond is important for cold activation of TRPM8, it is only shown in the presence of WS-12 and $\mathrm{PIP}_{2}$ but not observed in the presence of cholesterol hemisuccinate (CHS) only or in the absence of $\mathrm{PIP}_{2}$ (Figure 4A). ${ }^{[18,21]}$ Therefore, $\mathrm{PIP}_{2}$ is required for cold-evoked activation of TRPM8. The in silicon research demonstrates that $\mathrm{PIP}_{2}$ can bind to the interface between pre-S1 and TRP domains, promoting the gating coupling of the menthol pocket with the pore domain in pmTRPM8 in several ways (Figure 4B). First, the electrostatic interactions of the $\mathrm{C} 4$ phosphate moiety of $\mathrm{PIP}_{2}$ with the side chains of both $\mathrm{R} 679$ on pre-S1 and K988 in the TRP domain can stabilize edge-face $\pi-\pi$ interactions among aromatic rings of W673 on pre-S1 and F726/F729 on S1 and Y995 in the TRP domain. Second, those interfacial electrostatic interactions between $\mathrm{PIP}_{2}$ and pmTRPM8 rearrange S4 to disrupt the inhibitory R832D793 salt bridge for a release of the "parking brake". ${ }^{[23]}$ Third, those interfacial electrostatic interactions rotate the TRP helix in favor of the formation of the stimulatory R998-Q776 H-bond (Figure 4B). ${ }^{[19,21]}$

\subsection{The Putative Swapping Transition Metal Bridge between the VSLD and the Pore Domain is Required to Stablize the Cold Efficacy of TRPM8}

Although several cryo-EM structures of TRPM8 in the sensitized state are available, none is conducting cations through the channel pore. ${ }^{[18-19,21]}$ Since intracellular PH6 can suppress coldevoked TRPM8 activation, H835 on the voltage sensor $\mathrm{S} 4$, which is sensitive to acidic $\mathrm{pH}$ and included in the cold-dependent large hairpin formation with a 29-residue loop, may be involved. However, protonation of H835 cannot disconnect the H835-W789 interaction. Even if their $\pi-\pi$ interaction is weakened by protonation, a cation- $\pi$ interaction is still present between two aromatic residues. The in silicon investigation reveals that in the $\mathrm{Ca}^{2+}$-desensitized TRPM8, F858 on S5 is far away from W789 on S3. On the other hand, in the presence of low $\mathrm{Ca}^{2+}$ occupancy, even if $\mathrm{Ca}^{2+}$ can bind to E773 on S2 and T794 and N790 on S3 but leave Q776 free to form the stimulatory H-bond with R998, F858 is close to W789 but not enough to form a well-established face-face or edge-face $\pi-\pi$ interaction in the sensitized state (Figure $5 \mathrm{~A}$ ). The similar case was also found in the $\mathrm{Ca}^{2+}$-free closed state (Figure 5A). When WS-12 and PIP 2 are bound to TRPM8, even if the stimulatory R998-Q776 H-bond and the related large hairpin are available as a cold starter to allow a low temperature threshold to initiate TRPM8 activation, F858 is still not close enough to W789 to form a typical edge-face attractive $\pi-\pi$ interaction to stablize the cold efficacy (Figure 3 ). 
Therefore, protonation of H835 may disrupt the cold-evoked gating pathway of TRPM8 in another manner.

The cryo-EM structure of TRPM8 with WS-12 and PIP 2 bound indicates that M854 is close to F858 on S5 and H835 on S4 and W789 on S3 from one subunit, and N958 on S6 from the neighboring subunit (Figure 5A). Sequence alignment of the TRPM family shows that M854 is highly conserved (Figure 5B). It is also intriguing that both thioether ligation with methionine and strong cation- $\pi$ interactions with tryptophan can stabilize $\mathrm{Cu}(\mathrm{I})$ binding in protein $\mathrm{CusF}$. ${ }^{[28]}$ In this regard, it is possible that a strong interaction can be formed between a cationic metalthioether/imidazole center and the aromatic ring of W789 in the sensitized state. When such a transition metal ion is bound between H835 and W789 via a cation- $\pi$ interaction, EGTA at a concentration up to $10 \mathrm{mM}$ cannot remove it. ${ }^{[4,16,17,21,24-25,29]}$ Further, a dynamic swapping interaction between this metal site from one subunit and N958 on S6 from an adjacent subunit may turn S6 against S5 in favor of opening of both the upper and lower gates (Figure 5). ${ }^{[18]}$ Thus, this dynamic swapping interface between the VSLD and the pore domain may be required for a precise thermodynamic tuning of the cold-evoked TRPM8 activation.

When this putative transition metal site bridges W789 and H835 and M854 together, the smallest hairpin with no residue in the loop is born. What is more, in the desensitized TRPM8 with TC-I 2014 or AMTB bound, R998 in the TRP domain was found to H-bond with not only Q776 but also D772 on S2. In this case, the H-bonded R998 and Q776 and D772 may create another smaller hairpin with a 3-residue loop. Taken together, a putative hairpin with a 16-residue loop may be formed from Q851 to M854, W789, T794, Y784, W777, Q776, R998, Q987 so as to stablize the Q851-Q987 H-bond. However, when the putative transition metal bridge between the VSLD and the pore domain is missing, the TRPM8 channel is desensitized by those two antogonists. ${ }^{[18]}$ In any way, these smaller interfacial hairpins may be required to stabilize the coldevoked efficacy and to stop cold denaturation.

\section{DISCUSSION}

The TRP channels have a selectvity filter as the upper gate and the S6 bundle crossing as the lower gate to control the monvalent or divalent cation permeation throught the ion channel pore. Since these two gates are coupled to each other, opening of either gate can prime channel opening. The cryo-EM structural data show that the heat-sensing TRPV1 has a dynamic pore domain and a relatively static VSLD. ${ }^{[20]}$ In contrast, both the VSLD and the pore domain in the 
cold-sensing TRPM8 are dynamic. ${ }^{[30,18-19,21]}$ Thus, it is exciting to examine if temperaturedependent hairpin melting or formation along the gating pathway triggers activation of the thermosensitive TRP channels. When the heat-evoked melting of a larger hairpin in the outer pore was proposed to initiate TRPV1 activation, ${ }^{[31]}$ it is necessary to test if the cold-dependent formation of a larger hairpin in TRPM8 is responsible for the start of channel opening.

The lower S6 gate of the TRP channel is determined by the relative movement of the S4S5 linker against the VSLD or the TRP domain. The uplift of the S4-S5 linker can drive TRPV1 opening. ${ }^{[32]}$ However, little is known about how the lower S6 gate is opened by cold in TRPM8. Although the cryo-EM structures of TRPM8 were obtained in a frozen state, none is conducting $\mathrm{Ca}^{2+} .{ }^{[18-19,21]}$ Therefore, other experimental or in silicon approaches are needed to identify coldsensitive residues and the relevant gating pathway for design maps of TRPM8 as a thermometer.

Because capsaicin or its analog VBE can completely inhibit cold-evoked hTRPM8 currents and menthol-sensitive Y745 in the WS-12 or menthol or icilin pocket is involved, ${ }^{[21,23-24]}$ this menthol pocket may be intricate in the cold-initiated gating pathway of TRPM8. Since the molecular docking demonstrates that the short tail of VBE can disrupt the putative R998-Q776 Hbond when its vanillyl group H-bonds with the hydroxyl group of Y736 (Y745 in hTRPM8), the R998-Q776 H-bond may play a stimulatory role in the cold-evoked activation of TRPM8. In support of this proposal, $\mathrm{Ca}^{2+}$ binding can bridge E773 and Q776 on S2, Y784 in the S2-S3 linker, and D793 and N790 on S3 together to disrupt the putative stimulatory R998-Q776 H-bond for desensitization. ${ }^{[18,21]}$ As $\mathrm{Ca}^{2+}$ binding cannot change the temperature threshold of TRPM8, ${ }^{[25]}$ it is proposed that $\mathrm{Ca}^{2+}$ binding to E773 on S2 and T794 and N790 on S3 with low affinity may leave dynamic Q776 on S2 free to form the putative stimulatory H-bond with R998 in the TRP domain (Figure 5A). This affinity-dependent dynamic $\mathrm{Ca}^{2+}$ site is reminicant of the active and dynamic $\mathrm{Zn}^{2+}$ site at the $\mathrm{T}_{1}-\mathrm{T}_{1}$ interface of Kv4 channels. ${ }^{[33]}$ In the absence of $\mathrm{Ca}^{2+}$, the equivalent $\mathrm{H}$-bond between E773 on S2 and T794 or N790 on S3 may also favor the cold-evoked R998-Q776 H-bond for pmTRPM8 activation (Figure 5A). Once the R998-Q776 H-bond is formed, the Q851-Q987 H-bond between S5 and the TRP domain, together with the H835-W789 and Y784-W777 $\pi-\pi$ interactions and the Y784-T794 H-bond, produces a large hairpin with a 29-residue loop. This large hairpin loop starts from Q851 on S5 and links H835 on S5, W789 and T794 on S3, Y784 and W777 in the S2-S3 linker, Q776 on S2, R998 and Q987 in the TRP domain together for a low temperature threshold to initiate cold activation of TRPM8. Alternatively, when the D793-R832 
H-bond repalces the H835-W789 $\pi-\pi$ interaction, the similar hairpin size can be available (Figure 3).

On the other hand, extracellular acidic and alkaline $\mathrm{pH}$ can decrease and increase the temperature thresholds for cold activation of TRPM8, respectively. More importantly, intracellular pH6 can completely prohibit the cold-evoked activation of TRPM8. ${ }^{[10]}$ Thus, when acidic $\mathrm{pH}$-sensitive $\mathrm{H} 835$ in the large cold-dependent hairpin can form a face-face $\pi-\pi$ interaction with W789 on S3, both H835 and W789 can also sandwich a transition metal ion with a very high affinity via a cation- $\pi$ interaction. ${ }^{[29]}$ This putative transition metal cation may be $\mathrm{Zn}^{2+}, \mathrm{Cu}^{+}, \mathrm{Cu}^{2+}$, $\mathrm{Fe}^{2+}$, or $\mathrm{Fe}^{3+}$, depending on a different cellular redox environment. Previous studies demonstrated that these transition metal ions can bind to ion channels such as cystic fibrosis transmembrane conductance regulator (CFTR) and Slo $\mathrm{BK}_{\mathrm{Ca}}$ and $\mathrm{Kv} 4$ channels, regulating channel gating. ${ }^{29,33-}$ 39] Since TRPM8-expressing cells are mostly cultured in a $\mathrm{Fe}^{3+}$-containing media such as Dulbecco's modified Eagle's medium (DMEM) for cold-evoked electrophysiological experiments, it is more likely than not that the putative transition metal ion may be $\mathrm{Fe}^{2+}$ in the reducing enviroment. ${ }^{[29]}$ When the sandwiched transition metal cation interacts with M854 on S5, the additional swaping dynamic interaction between this metal site from one subunit and N958 on S6 from another adjacent subunit may facilitate the relative movement of the pore domain against the VSLD and the TRP domain in favor of opening of both upper and lower gates in the channel pore. Thus, the putative transition metal bridge of M854 on S5 with H835 on S4 and W789 on S3, together with the H-bonded R998 in the TRP domain and D772 and Q776 on S2, can prepare two smaller hairpins with 0 to 3-residue loops to stabilize the cold efficacy and to avoid cold denaturation (Figure 3).

The ligand-free cryo-EM structure of pmTRPM8 indicates that the stimulatory R998-Q776 H-bond is absent to drive TRPM8 opening. ${ }^{[18-19,21]}$ On the other hand, high concentrations of $\mathrm{PIP}_{2}$ can increase the temperature threshold from $20-28^{\circ} \mathrm{C}$ to $32-37^{\circ} \mathrm{C}$ for TRPM8 activation under naive conditions, but depletion of $\mathrm{PIP}_{2}$ by poly-lysine can completely stop cold-evoked TRPM8 activation. ${ }^{[5]}$ Thus, $\mathrm{PIP}_{2}$ is required for cold-driven TRPM8 opening. In sharp contrast, the release of the resident phosphatidylinositol lipid in the vanilloid site is necessary for heat or vanilloid compouds to activate TRPV1. ${ }^{[31,40]}$

The orientation of the side chain of R998 in pmTRPM8 is important for the putative stimulatory R998-Q776 H-bond. The cryo-EM structures of pmTRPM8 denote that R998 (R1008 
in mTRPM8 or hTRPM8) cannot form a stable H-bond with Q776 (Q785 in mTRPM8 or hTRPM8) in the absence of lipid bound between the TRP domain and the pre-S1 domain. When $\mathrm{PIP}_{2}$ interacts with R988 in the TRP domain and K679 and N683 in the pre-S1 domain, a rotation of the TRP domain may pull Y995 close to F729 on S1 and W673 on pre-S1 to form attractive edge-face $\pi-\pi$ interactions as a stable anchor in support of the putative stimulatory R998-Q776 Hbond. That's why high concentration of $\mathrm{PIP}_{2}$ alone can activate TRPM8 at a warm temperature (Figures 3-4). Regarding the exposed-temperature-dependent changes in temperature thresholds of hTRPM8 upon the allosteric interaction of $\mathrm{PIP}_{2}$ with R1008 (R998 in pmTRPM8), ${ }^{[25]}$ it is possible that the presence of K995 (K985 in pmTRPM8) or R998 (R988 in pmTRPM8) in the TRP helix, and R688 (R679 in pmTRPM8), or K691 (K682 in pmTRPM8) or K694 (K685 in pmTRPM8) in the pre-S1 helix may allow $\mathrm{PIP}_{2}$ to serve as a gear to interact with different positive residues between the TRP domain and the pre-S1 domain to tune the intensity of the putative R1008-Q785/D781 (R998-Q776/D772 in pmTRPM8) H-bonds for different thresholds in response to varied ambient temperatures (Figure 5B).

Since $\mathrm{Ca}^{2+}$ binding with a high affinity between $\mathrm{S} 2$ and S3 can desensitize the mentholevoked activation, ${ }^{[18,23]}$ when the putative H-bonded homochiral menthol dimer pushes the side chain of Y995 (Y1005 in hTRPM8) in the TRP helix away from the lower S6 bundle crossing gate against S4, it may bring R998 (R1008 in hTRPM8) in the same TRP helix close to Q776 (Q785 in hTRPM8) to form the stimulatory H-bond for TRPM8 activation. That's why menthol can elevate the temperation threshold of TRPM8 for cold activation at $\mathrm{pH} 7.4$ via the same gating pathway. ${ }^{[1-}$ 2, 10, 41] As both menthol and $\mathrm{PIP}_{2}$ can reposition the TRP domain in different ways for the stimulatory equivalent R998-Q776/D772 H-bond, it is reasonable that both cold and menthol can increase the apparent affinity of the TRPM8 channel for $\mathrm{PIP}_{2 .}{ }^{[5]}$ In this regard, the stimulatory R998-Q776/D772 H-bond may also be indispensable for TRPM8 activation by the H-bonded homochiral menthol dimer. ${ }^{[23]}$

The TRPM family, part of the TRP channel superfamily, has eight members (TRPM1 to TRPM8). In addition to cold-sensitive TRPM8 $\left(<20-28{ }^{\circ} \mathrm{C}\right)$, cold to warm-sensitive TRPM channels include TRPM2 $\left(>36^{\circ} \mathrm{C}\right)$, TRPM4 and TRPM5 $\left(>22^{\circ} \mathrm{C}\right)$ but TRPM3 is a sensor for noxious heat. ${ }^{[1,42-45]}$ Sequence alignment of the TRPM family indicates that some highly conserved residues may be important for cold to warm sensitive TRPM channels. While the equivalent stimulatory R998-Q776/D772 H-bonds are almost conserved among all the TRPM 
family, the putative dynamic swapping transition metal site with very high affinity may be only highly conserved among human TRPM2, TRPM4, TRPM5 and TRPM8 but not in human TRPM67 and TRPM1 and TRPM3 (Figure 5B). Accordingly, both the R998-Q776/D772 H-bonds and the N958-Metal-M854/H835/W789 bridge are indispensable and coupled together as a team for the cold-evoked activation of TRPM8.

\section{CLOSING REMARK}

The TRPM8 channel can be activated by both cold and cooling agents such as menthol and its analog WS-12. Since the menthol pocket is required for cold-activation, both physical and chemical stimuli may share the same gating pathway although residues sensitive to cold and menthol may be different. While the putative $\mathrm{Ca}^{2+}$-independent $\mathrm{H}$-bond between the $\mathrm{Ca}^{2+}$ site in VSLD and the C-terminal of the TRP helix is responsible for the larger hairpin formation to ensure the low temperature threshold, the putative dynamic swapping interfacial transition metal bridge between the VSLD and the pore domain, together with $\mathrm{Ca}^{2+}$-independent $\mathrm{H}$-bonds between VSLD and the TRP helix, is needed to form the smallest hairpins to stabilize the cold efficacy and to prevent cold denaturation. The cold-induced global hairpin rearrangements along the gating pathway from the dynamic interface between the VSLD and the TRP domain to the dynamic interface between the VSLD and the pore domain may prime the high temperature coefficient or Q10. Taken all these into account, this study may further support the hypothesis regarding the temperature-dependent topological hairpin structures for thermosensitive TRP channels.

\section{MATERIALS AND METHODS}

In this in silicon study, the hairpin topological structural aspects of TRPM8 channels in different states at low temperature were investigated and analyzed with graph theory as the thermometer basis for noxious cold detection. The in silico protomer models were based on the cryo-EM structural data of closed pmTRPV1 with CHS-bound (PDB ID, 6O6A) and additional $\mathrm{Ca}^{2+}$-bound (PDB ID, 6O77), ${ }^{[18]}$ sensitized cfTRPM8 with WS-12 and PIP 2 bound (PDB ID, 6NR2), desensitized cfTRPM8 with icilin and $\mathrm{Ca}^{2+}$ and $\mathrm{PIP}_{2}$ bound (PDB ID, 6NR3), and denatured cfTRPM8 without PIP 2 bound (PDB ID, 6BPQ). ${ }^{[19,21]}$ UCSF Chimera was used for molecular docking and topological hairpin analyses.

For molecular docking, the different rotamers of the residues in the menthol pocket of TRPM8 were tested to optimally interact with the introduced capsaicin or VBE. The different orientations of the test molecules were also examined to make sure that their spatial hindrance in 
the pocket is minimal but their non-covalent interactions with nearby residues in the pocket are maximal.

For topological hairpin analyses, all the potential non-covalent interactions such as salt and metal bridges and $\pi-\pi$ or cation- $\pi$ interactions and H-bonds along the gating pathway in TRPM8 were included to examine their possible roles in forming the smallest hairpins, controlling the temperature threshold and the temperature sensitivity Q10, and stablizing the cold afficacy. The smallest hairpin sizes to govern the thermostability of non-covalent interactions were calculated using graph theory. The different residues for activation of TRPM8 by cold and the cooling agents such as menthol were also compared. Q10 was used to characterize the temperature-dependent responses as calculated using the following equation:

$$
\mathrm{Q}_{10}=(\mathrm{R} 2 / \mathrm{R} 1)^{10 /(\mathrm{T} 2-\mathrm{T} 1)}
$$

Conventions and Abbreviations: CHS, cholesterol hemisuccinate; CFTR, cystic fibrosis transmembrane conductance regulator; cryo-EM, cryo-electron microscopy; DMEM, Dulbecco's modified Eagle's medium; HTH, helix-turn-helix; PIP2, phosphatidylinositol-4,5-bisphosphate; TRP, transient receptor potential; TRPM8, TRP melastatin-8; cfTRPM8, collared flycatcher TRPM8; hTRPMi, human TRPMi (i=1, 2, 3, 4, 5, 6, 7, 8); mTRPM8, mouse TRPM8; pmTRPM8, Parus major TRPM8; rTRPM8, rat TRPM8; TRPV1, TRP vanilloid-1; VBE, Vanillyl butyl ether; VSLD, voltage-sensor-like domain

\section{Acknowledgements}

The author's own studies cited in this article were supported by the American Heart Association Grant (10SDG4120011 to GW), National Institute of Health Research Grants R01 NS032337, P01 N037444, T32 AA07463, DK45880, and 2R56DK056796-10, and Cystic Fibrosis Foundation grant (DAWSON0210).

Conflict of Interest: The author declares no conflict of interest.

Data Availability Section: This study includes no data deposited in external repositories. 


\section{References}

1. D. D. McKemy, W. M. Neuhausser, D. Julius. Identification of a cold receptor reveals a general role for TRP channels in thermosensation. Nature 2002, 416, 52.

2. A. M. Peier, A. Moqrich, A. C. Hergarden, A. J. Reeve, D. A. Andersson, G. M. Story, T. J. Earley, I. Dragoni, P. McIntyre, S. Bevan, A. Patapoutian. A TRP channel that senses cold stimuli and menthol. Cell 2002, 108, 705.

3. G. Reid, A. Babes, F. Pluteanu. A cold- and menthol-activated current in rat dorsal root ganglion neurones:properties and role in cold transduction. J Physiol (Lond), 2002, 545, 595.

4. H. H. Chuang, W. M. Neuhausser, D. Julius, The super-cooling agent icilin reveals a mechanism of coincidence detection by a temperature-sensitive TRP channel. Neuron, 2004, 43,859 .

5. T. Rohacs, C. M. B. Lopes, I. Michailidis, D. E. Logothetis. PI(4,5)P2 regulates the activation and desensitization of TRPM8 channels through the TRP domain. Nat Neurosci. 2005, 8, 626.

6. B. Liu, F. Qin. Functional control of cold- and menthol-sensitive TRPM8 ion channels by phosphatidylinositol 4, 5-bisphosphate. J Neurosci. 2005, 25, 1674.

7. T. Voets, G. Owsianik, A. Janssens, K. Talavera, B. Nilius. TRPM8 voltage sensor mutants reveal a mechanism for integrating thermal and chemical stimuli. Nat Chem Biol. 2007, 3, 174.

8. M. Bodding, U. Wissenbach, V. Flockerzi. Characterisation of TRPM8 as a pharmacophore receptor. Cell Calcium 2007, 42, 618.

9. E. Zakharian, C. Cao, T. Rohacs. Gating of transient receptor potential melastatin 8 (TRPM8) channels activated by cold and chemical agonists in planar lipid bilayers. J Neurosci. 2010, $30,12526$.

10. D. A. Andersson, H. W. Chase, S. Bevan. TRPM8 activation by menthol, icilin, and cold is differentially modulated by intracellular pH. J Neurosci, 2004, 24, 5364.

11. T. Voets, G. Droogmans, U. Wissenbach, A. Janssens, V. Flockerzi, B. Nilius. The principle of temperature-dependent gating in cold- and heat-sensitive TRP channels. Nature 2004, 430, 748.

12. D. M. Bautista, J. Siemens, J. M. Glazer, P. R. Tsuruda, A.I. Basbaum, C. L. Stucky, S. E. Jordt, D. Julius. The menthol receptor TRPM8 is the principal detector of environmental cold. Nature 2007, 448, 204. 
13. R. W. Colburn, M. L. Lubin, D. J. Stone Jr., Y. Wang, D. Lawrence, M. R. D’Andrea, M. R. Brandt, Y. Liu, C. M. Flores, N. Qin. Attenuated cold sensitivity in TRPM8 null mice. Neuron 2007, 54, 379 .

14. A. Dhaka, A. N. Murray, J. Mathur, T. J. Earley, M. J. Petrus, A. Patapoutian. TRPM8 is required for cold sensation in mice. Neuron 2007, 54, 371.

15. W. M. Knowlton, A. Bifolck-Fisher, D. M. Bautista, D. D. McKemy. TRPM8, but not TRPA1, is required for neural and behavioral responses to acute noxious cold temperatures and coldmimetics in vivo. Pain, 2010, 150, 340.

16. S. Wang, J. Lee, J. Y. Ro, M. K. Chung. Warmth suppresses and desensitizes damage-sensing ion channel TRPA1. Mol Pain. 2012, 8, 22.

17. M. Bandell, A. E. Dubin, M. J. Petrus, A. Orth, J. Mathur, S. W. Hwang, A. Patapoutian. High-throughput random mutagenesis screen reveals TRPM8 residues specifically required for activation by menthol. Nat. Neurosci. 2006, 9, 493.

18. M. M. Diver, Y. Cheng, D. Julius. Structural insights into TRPM8 inhibition and desensitization. Science 2019, 365, 434.

19. Y. Yin, M-Y. Wu, L. Z. Zubcevic, W. F. Borschel, G. C. Lander, S-Y Lee. Structure of the cold- and menthol-sensing ion channel TRPM8. Science 2018, 359, 237.

20. K. Zhang, D. Julius, Y. Cheng. Structural snapshots of TRPV1 reveal mechanism of polymodal functionality. Cell 2021, 184, 1.

21. Y. Yin, S. C. Le, A. L. Hsu, M. J. Borgnia, H-Y. Yang, S-Y Lee. Structural basis of cooling agent and lipid sensing by the cold-activated TRPM8 channel. Science 2019, 363, 237.

22. L. Xu, Y. Han, X. Chen, A. Aierken, H. Wen, W. Zheng, H. Wang, X. Lu, Z. Zhao, C. Ma, P. Liang, W. Yang, S. Yang, F. Yang. Molecular mechanisms underlying menthol binding and activation of TRPM8 ion channel. Nat Commun. 2020, 11, 3790.

23. G. Wang. Ligand-stereoselective allosteric activation of cold-sensing TRPM8 channels by an H-bonded homochiral menthol dimer with head-to-head or head-to-tail. Chirality 2021, 33, 783.

24. M. Takaishi, K. Uchida, Y. Suzuki, H. Matsui, T. Shimada, F. Fujita, M. Tominaga. Reciprocal effects of capsaicin and menthol on thermosensation through regulated activities of TRPV1 and TRPM8. J Physiol Sci., 2016, 66, 143. 
25. F. Fujita, K. Uchida, M. Takaishi, T. Sokabe, M. Tominaga. Ambient temperature affects the temperature threshold for TRPM8 activation through interaction of phosphatidylinositol 4, 5bisphosphate. J Neurosci. 2013, 33, 6154.

26. A. T. Jonstrup, J. Fredsøe, A. H. Andersen, DNA Hairpins as Temperature Switches, Thermometers and Ionic Detectors. Sensors 2013, 13, 5937.

27. R. L. Daniels, Y. Takashima, D. D. McKemy. Activity of the neuronal cold sensor TRPM8 is regulated by phospholipase $\mathrm{C}$ via the phospholipid phosphoinositol 4,5-bisphosphate. $J$ Biol Chem. 2009, 284, 1570.

28. Y. Xue, A. V. Davis, G. Balakrishnan, J. P. Stasser, B. M. Staehlin, P. Focia, T. G. Spiro, J. E. Penner-Hahn, T. V. O’Halloran. $\mathrm{Cu}(\mathrm{I})$ recognition via cation- $\pi$ and methionine interactions in CusF. Nat Chem Biol. 2008, 4, 107.

29. G. Wang. Mechanistic insight into the heme-independent interplay between iron and cartbon monoxide in CFTR and Slo1 BKca channels. Metallomics 2017, 9, 634.

30. M. Pertusa, B. Rivera, A. González, G. Ugarte, R. Madrid. Critical role of the pore domain in the cold response of TRPM8 channels identified by ortholog functional comparison. $J$ Biol Chem. 2018, 293, 12454.

31. G. Wang. Heat-dependent hairpin melting drives TRPV1 opening. bioRxiv 2022 (doi.org/10.1101/2022.01.02.474701).

32. D. H. Kwon, F. Zhang, Y. Suo, J. Bouvette, M. J Borgnia, S-Y. Lee. Heat-dependent opening of TRPV1 in the presence of capsaicin. Nat Struct Mol Biol. 2021, 28, 554.

33. G. Wang, C. Strang, P. J. Pfaffinger, M. Covarrubias. $\mathrm{Zn}^{2+}$-dependent redox switch in the intracellular $\mathrm{T}_{1}-\mathrm{T}_{1}$ interface of a Kv Channel. J. Biol. Chem. 2007, 282, 13637.

34. X. Liu, C. Alexander, J. Serrano, E. Borg, D. C. Dawson. Variable Reactivity of an Engineered Cysteine at Position 338 in Cystic Fibrosis Transmembrane Conductance Regulator Reflects Different Chemical States of the Thiol. J. Biol. Chem. 2006, 281, 8275.

35. G. Wang. State-dependent regulation of cystic fibrosis transmembrane conductance regulator (CFTR) gating by a high affinity $\mathrm{Fe}^{3+}$ bridge between the regulatory domain and cytoplasmic loop 3. J Biol Chem. 2010, 285, 40438.

36. G. Wang. Interplay between inhibitory ferric and stimulatory curcumin regulates phosphorylation-dependent human cystic fibrosis transmembrane conductance regulator and $\Delta$ F508 activity. Biochemistry 2015, 54, 1558. 
37. G. Wang. Molecular Basis for Fe(III)-Independent Curcumin Potentiation of Cystic Fibrosis Transmembrane Conductance Regulator Activity. Biochemistry 2015, 54, 2828.

38. G. Wang, R. Linsley, Y. Norimatsu. External $\mathrm{Zn}^{2+}$ binding to cysteine-substituted cystic fibrosis transmembrane conductance regulator constructs regulates channel gating and curcumin potentiation. FEBS J. 2016, 283, 2458.

39. G. Wang. Removal of the Fe(III) site promotes activation of the human cystic fibrosis transmembrane conductance regulator by high-affinity $\mathrm{Zn}(\mathrm{II})$ binding. Metallomics 2018,10, 240.

40. G. Wang. Lipid-dependent sequential allosteric activation of heat-sensing TRPV1 channels by anchor-stereoselective "hot" vanilloid compounds and analogs. Biochem. Biophys. Rep. 2021, 28,101109.

41. A. Mälkiä, R. Madrid, V. Meseguer, E. De la Peña, M. Valero, C. Belmonte, F. Viana. Bidirectional shifts of TRPM8 channel gating by temperature and chemical agents modulate the cold sensitivity of mammalian thermo-receptors. J Physiol. 2007, 581,155.

42. K. Talavera, K. Yasumatsu, T. Voets, G. Droogmans, N. Shigemura, Y. Ninomiya, R. F. Margolskee, B. Nilius. Heat activation of TRPM5 underlies thermal sensitivity of sweet taste. Nature 2005, 438, 1022.

43. J. Vriens, G. Owsianik, T. Hofmann, S. Philipp, J. Stab, X. Chen, M. Benoit, F. Xue, A. Janssens, S. Kerselaers, J. Oberwinkler, R. Vennekens, T. Gudermann, B. Nilius, T. Voets. TRPM3 is a nociceptor channel involved in the detection of noxious heat. Neuron 2011, 70, 482.

44. K. Song, H. Wang, G. B. Kamm, J. Pohle, F. C. Reis, P. Heppenstall, H. Wende, J. Siemens. The TRPM2 channel is a hypothalamic heat sensor that limits fever and can drive hypothermia. Science 2016, 353,1393.

45. C. H. Tan, P. A. McNaughton. The TRPM2 ion channel is required for sensitivity to warmth. Nature 2016, 536, 460. 


\section{Figure legends}

Figure 1 The hairpin test model for examining the cold-evoked gating pathway of TRPM8 channels. Non-covalent interactions such as salt and metal bridges and aromatic side chain $\pi-\pi$ interactions and H-bonds in the TRPM8 channel are proposed to form hairpins with different loop sizes. Low and high temperature thresholds allow hairpins with the large and small loops to form, respectively. The physical and chemical stimuli including ionic strength on the hairpin loop can regulate the loop plasticity for different temperature thresholds. Only the hairpin opening and closure along the gating pathway may govern the temperature threshold and cold efficacy for TRPM8 activation.

Figure 2. The non-covalent interactions (A) and the hairpins map (B) of $\mathrm{Ca}^{2+}$-desensitized TRPM8 along the gating pathway. A, The non-covalent interactions were based the cryo-EM structure of pmTRPM8 with $\mathrm{Ca}^{2+}$ bound (PDB ID, 6O77). ${ }^{[18]}$ Salt and metal bridges and H-bonds and $\pi-\pi$ interactions enhance the intra- and inter-domain interactions along the gating pathway. The residues are colored in red for a positive charge or blue for a negative charge in salt bridges or $\mathrm{H}$ bonds, dark blue for metal bridges, orange for the uncharged residues in H-bonds, and green for $\pi-\pi$ interactions. The aromatic pairs for $\pi-\pi$ interactions are mainly clustered in the VSLD and its interfaces with the pre-S1 domain and the TRP domain. B, The pore domain, the S4-S5 linker, the TRP domain, the VSLD and the pre-S1 domain are indicated. Salt bridges, the metal bridge, $\pi-\pi$ interactions, and $\mathrm{H}$-bonds are marked in purple, dark blue, green and orange, respectively. The smallest hairpin loop sizes required to control the non-covalent interactions were calculated using graph theory and labeled.

Figure 3. The non-covalent interactions (A) and the hairpins map (B) of sensitized TRPM8 along the gating pathway in the presence of WS-12 and PIP $_{2}$. A, The non-covalent interactions in pmTRPM8 were based on the cryo-EM structure of sensitized cfTRPM8 in the presence of WS12 and $\mathrm{PIP}_{2}$ (PDB ID, 6NR2). ${ }^{[21]}$ Salt bridges and H-bonds and $\pi-\pi$ interactions enhance the intra- and inter-domain interactions along the gating pathway. The residues are colored in red for a positive charge or blue for a negative charge in salt bridges or $\mathrm{H}$-bonds, orange for the uncharged residues in H-bonds, and green for $\pi-\pi$ interactions. The aromatic pairs for $\pi-\pi$ interactions are mainly clustered in the VSLD and its interfaces with the pre-S1 domain and the TRP domain. B, 
The pore domain, the S4-S5 linker, the TRP-like domain, the VSLD and the pre-S1 domain are indicated. Salt bridges, $\pi-\pi$ interactions and H-bonds are marked in purple, green and orange, respectively. The dashed lines are putative non-covalent interactions for cold activation of TRPM8. The smallest hairpin loop sizes required to control the non-covalent interactions were calculated using graph theory and labeled. The putative loop sizes are marked in grey.

Figure 4. $\mathrm{PIP}_{2}$ is required for the stimulatory R998-Q776 H-bond in TRPM8. A, Non-covalent interactions of TRPM8 in the menthol pocket in different gating states. The in silicon protomer models were based on the cryo-EM structural data of pmTRPM8 or cfTRPM8 in the closed (PDB ID: 6O6A), sensitized (PDB ID: 6NR2 or 6NR4), desensitized (PDB ID, 6NR3) and denatured (PDB ID, 6BPQ) states. ${ }^{[18,21]}$ The reposition of the TRP domain against the VSLD faciliates the lower S6 gate opening. When the temerature is lowered below a threshold, PIP $_{2}$ can bridge R679 and N683 on pre-S1 and R988 in the TRP domain and K596 from the adjacent subunit, stablizing the aromatic edge-face $\pi-\pi$ interactions of Y995 in the TRP domain with both F729 on S1 and W673 on pre-S1 in favor of the stimulatory side chain H-bond between R998 in the TRP domain and Q776 on S2. On the other hand, either $\mathrm{Ca}^{2+}$ binding between S2 and S3 or VBE binding with the tail between R998 and Q776 may break the stimulatory R998-Q776 H-bond to desensitize or to inactivate the TRPM8 channel, respectively.

Figure 5 The trace transition metal ion in the swapping dynamic interface between the VSLD and the pore domain of TRPM8 is required for cold-evoked efficacy. A, The effects of intracellular $\mathrm{Ca}^{2+}$ and transition metal ions on the gating states of TRPM8. The different gating states were based cryo-EM structures of TRPM8: closed (PDB ID, 6O6A), sensitized (PDB ID, 6NR2 or 6NR4), desensitized (PDB ID, 6NR3) and open (PDB ID, 6NR2). ${ }^{[18,21]}$ Intra-subunit $\mathrm{Ca}^{2+}$ binding to E773 on S2 and N790 and T794 on S3 can sensitize TRPM8 at low occupancy for the stimulatory R998-Q776 H-bond. However, Ca ${ }^{2+}$ binding to E773 and Q776 on S2 and N790 and D793 on S3 and Y784 in the S2-S3 linker at high occupancy desensitizes TRPM8 by disrupting the R998-Q776 H-bond. On the other hand, a putative inter-subunit transition metal site is required to glue swapped S5 and S6 close together to open the lower and upper gates simutaneously. The metal binding ligands include W789 on S3 and H835 on S4 and M854 on S5 from one subunit, and N958 on S6 from the neighboring subunit. The $\mathrm{Ca}^{2+}$ binding with high affinity seperates M854 
away from W789 and H835 to prevent the stimulatory and dynamic and interfacial swapping metal bridge with N958. B, Sequence alignment of TRPM channel family members. Residues discussed in the text are bolded and colored. Numbers above and below the sequences refer to mTRPM8 and hTRPM1 channels, respectively. The residues are colored in red for a positive charge or blue for a negative charge in salt bridges or H-bonds, orange for the uncharged residues in H-bonds, green for $\pi-\pi$ interactions, dark blue for metal bridges. N958 is from the neighboring subunit and marked in different blue. The upper non-covalent interactions favor the open state while the lower ones desensitize TRPM8. 


\section{Figures}

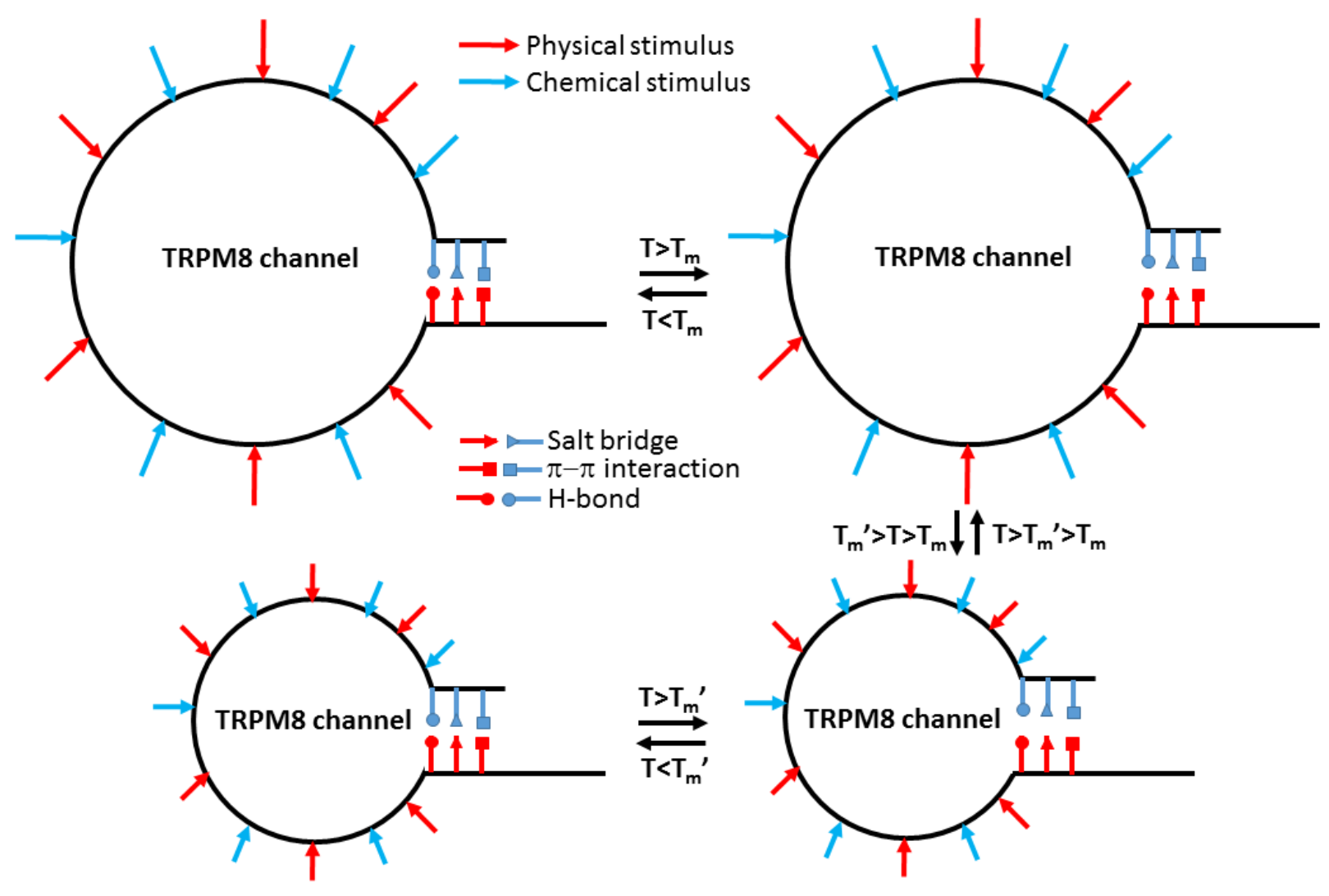

Figure 1 
A
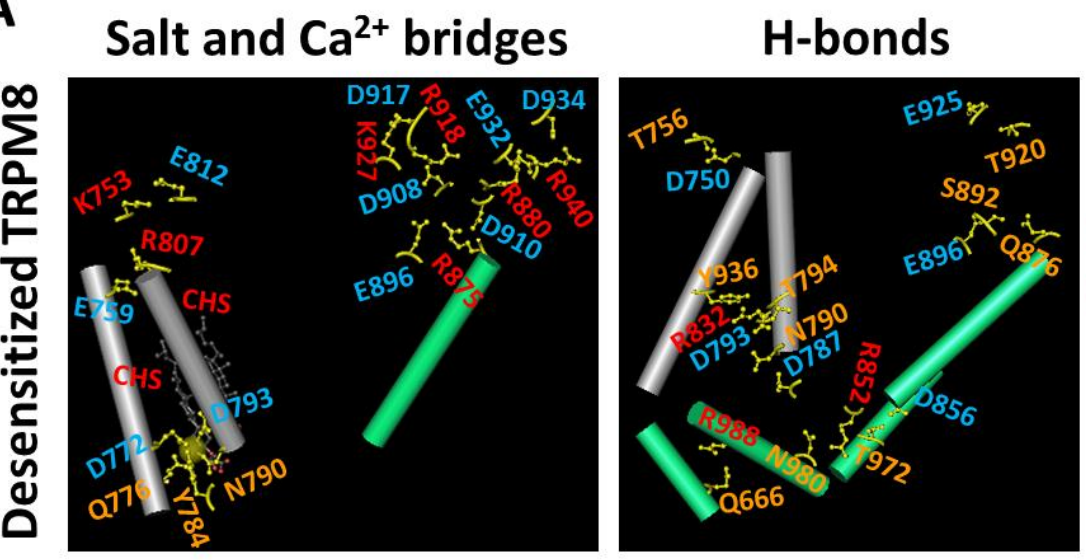

$\pi-\pi$ interactions

B

Desensitized TRPM8 with $\mathrm{Ca}^{2+}$ and CHS bound

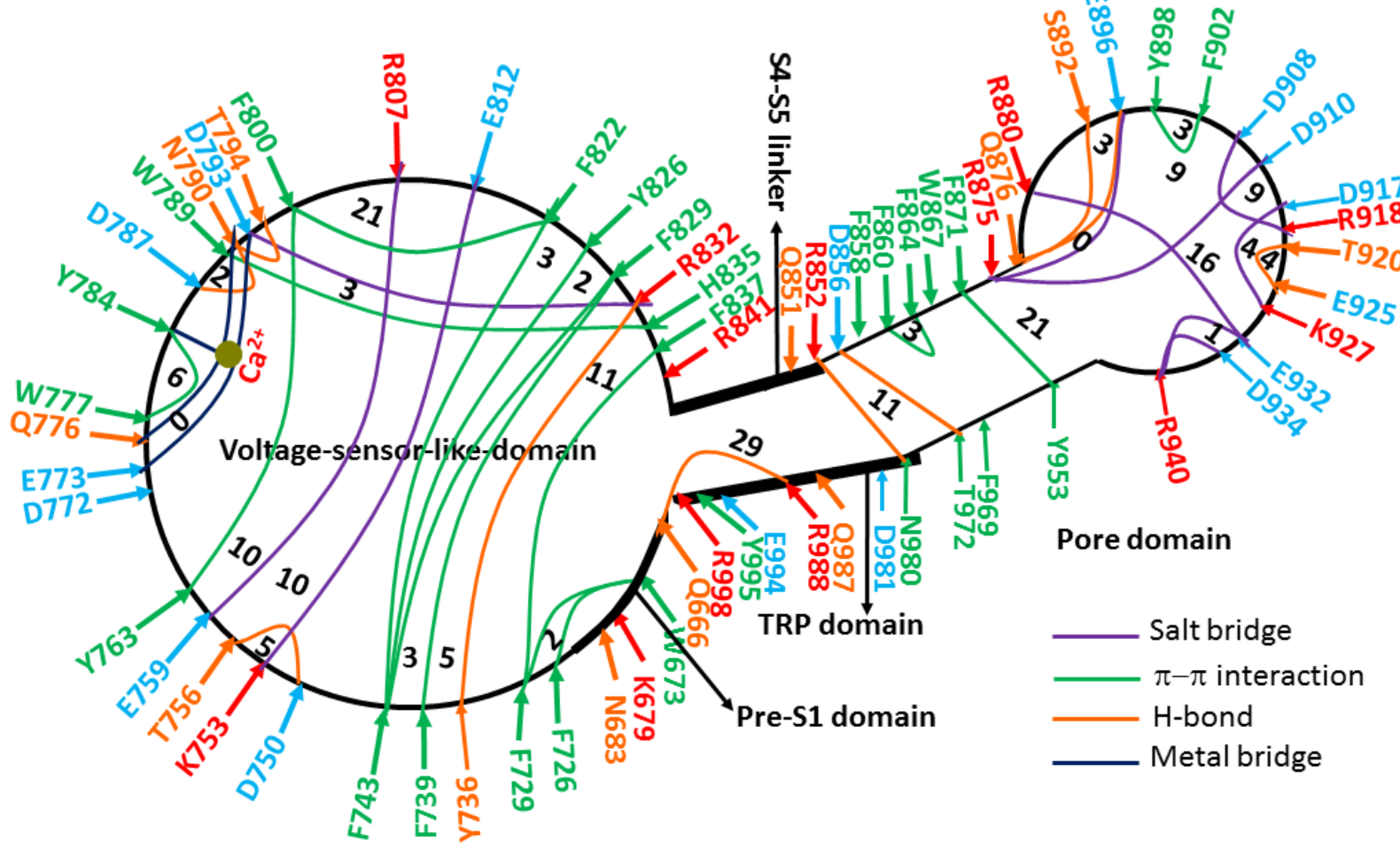

Figure 2 
A

Salt bridges

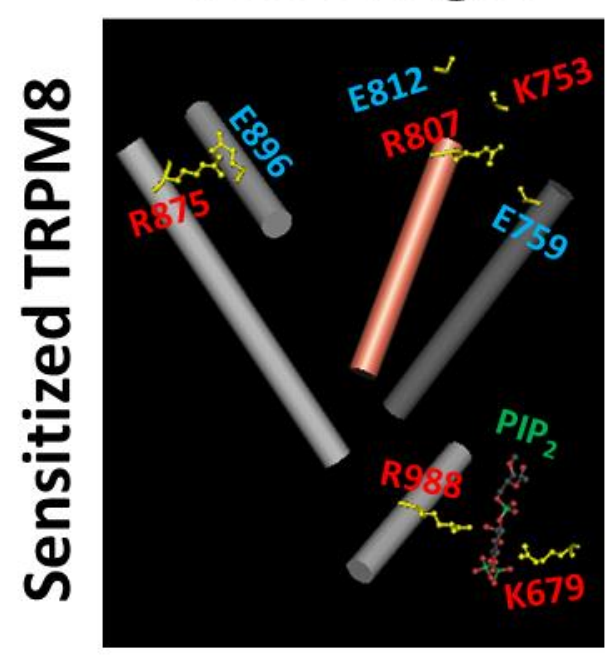

H-bonds

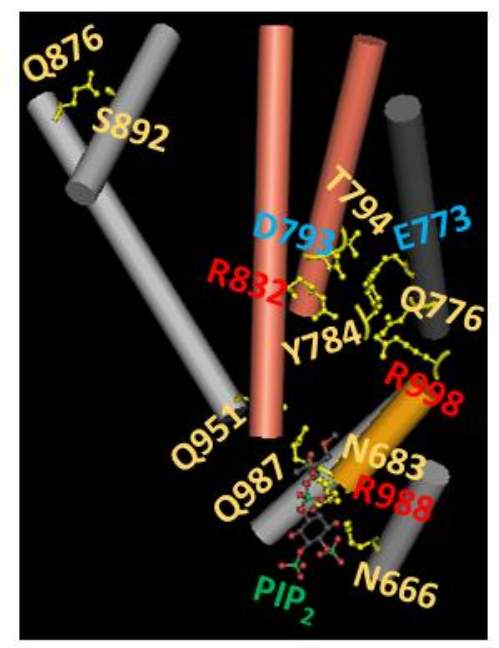

$\pi-\pi$ interactions

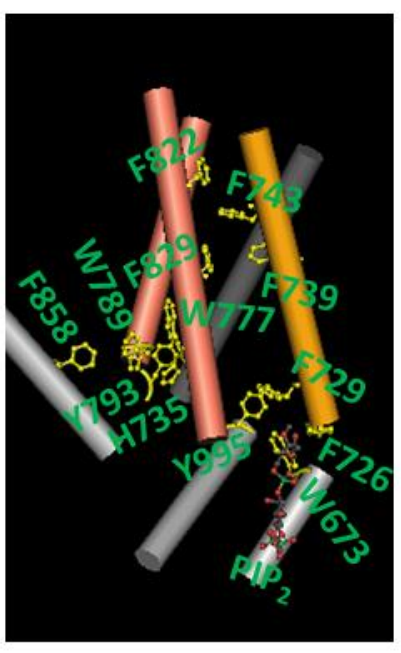

B

Sensitized TRPM8 with WS-12 and PIP $_{2}$ bound

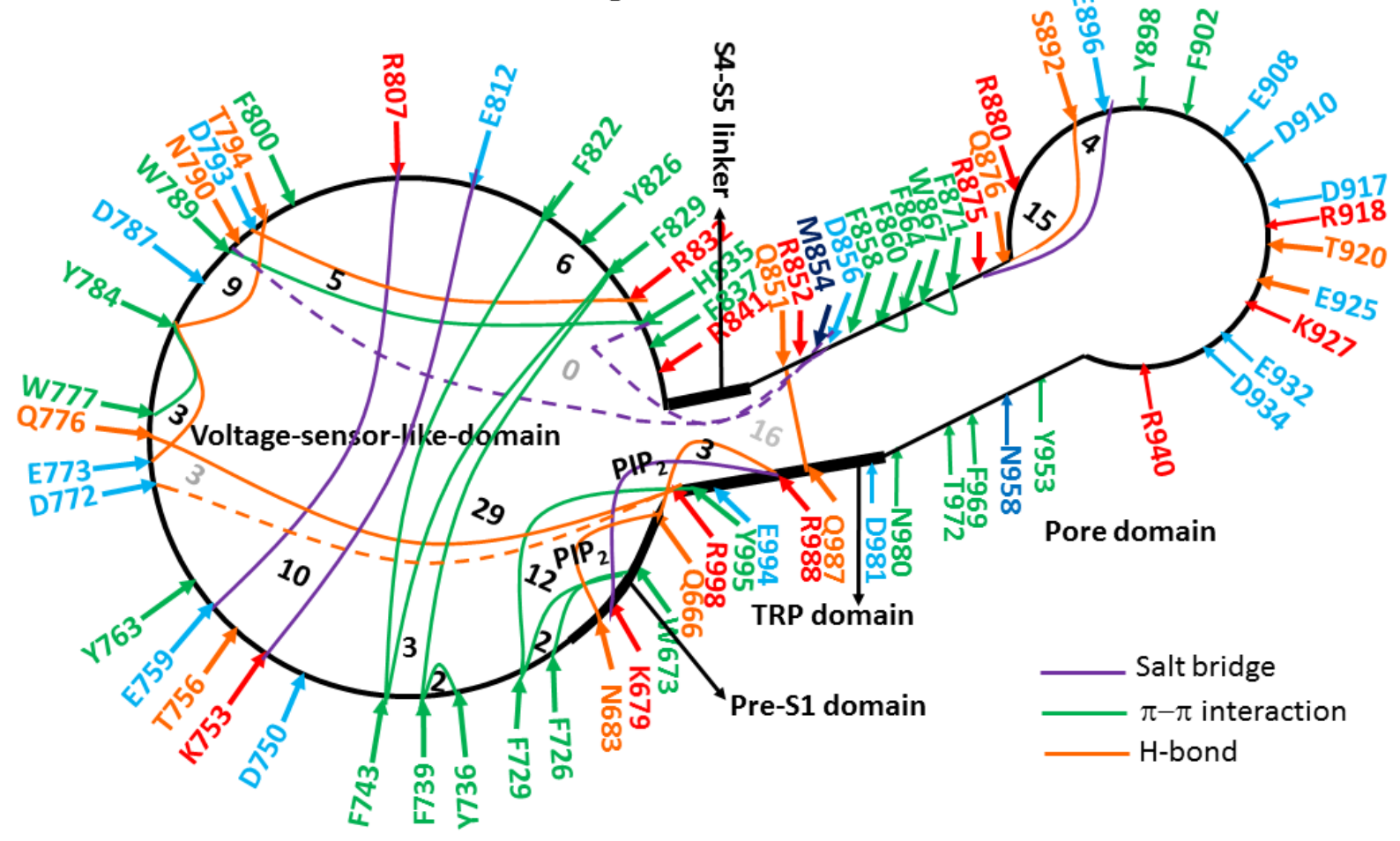

Figure 3 


\section{A}
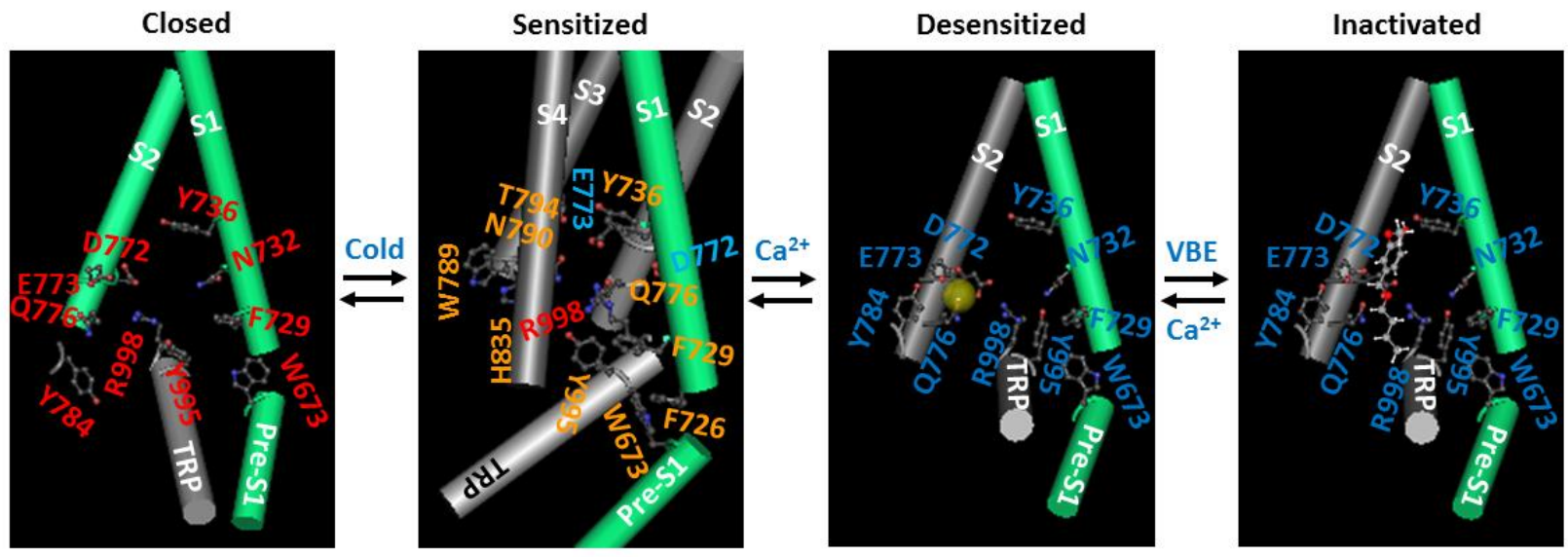

\section{B}
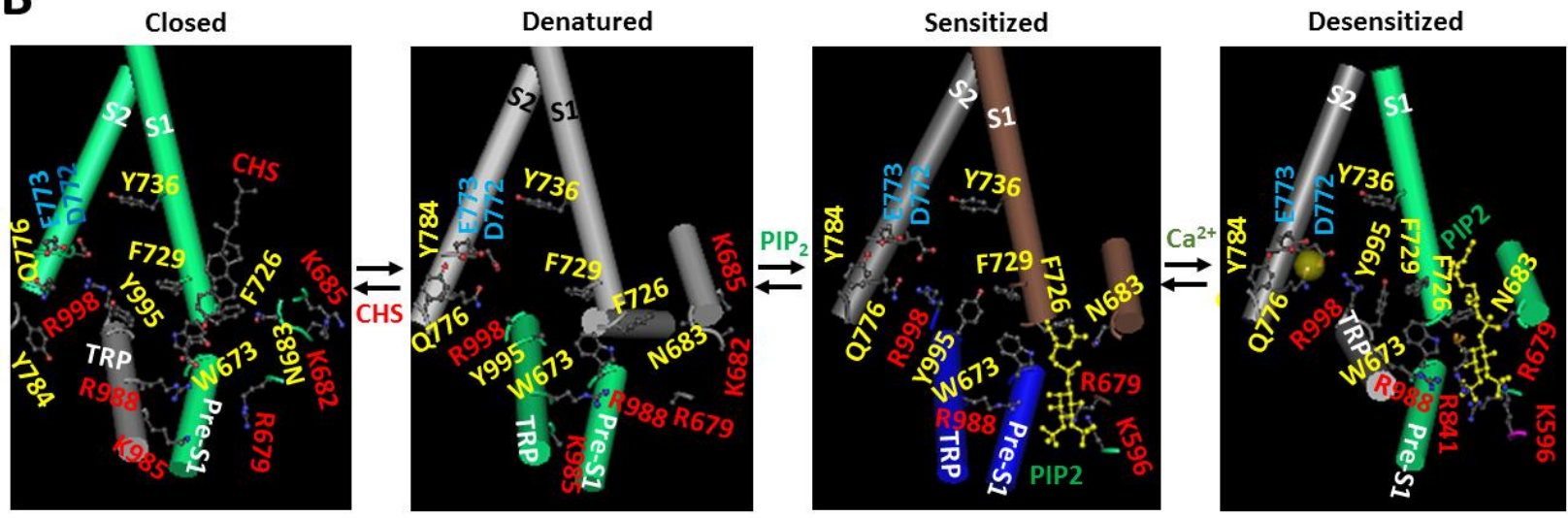

Figure 4 
bioRxiv preprint doi: https://doi.org/10.1101/2022.01.02.474742; this version posted January 12, 2022. The copyright holder for this preprint (which was not certified by peer review) is the author/funder. All rights reserved. No reuse allowed without permission.

A

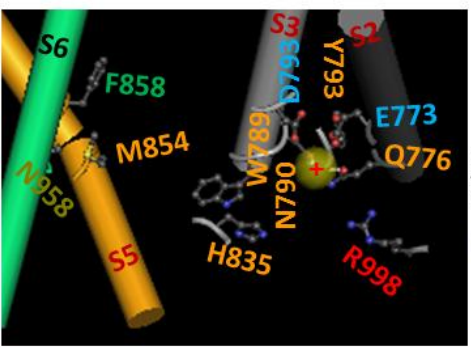

Desensitized

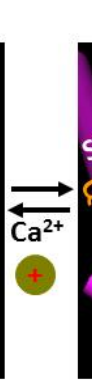

Sensitized

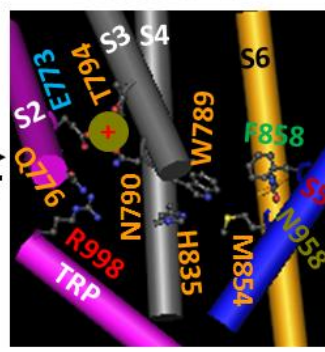

Closed

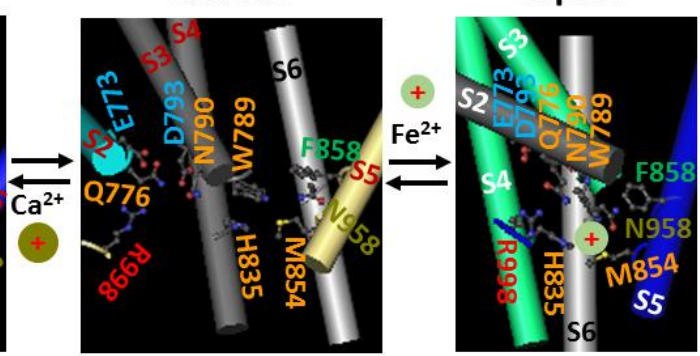

B

PIP2 bridge (gear)

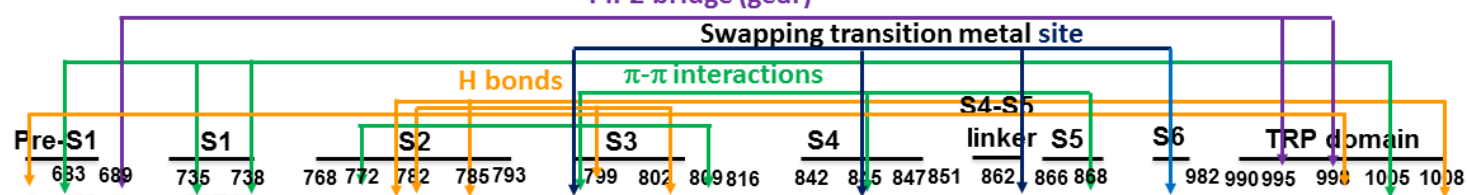

mTRPM8 $675 \mathrm{Q}$ WY R $735 \mathrm{FVVF} 768 \mathrm{E}$ Y DEVRQ Y 798 WNVMDT F R 842 RLIHIF $R$ ORM D F $968 \underline{N}$ T_N_KEOR EYCNR pmTRPM8 666 Q WY R 726 FVVF $759 \mathrm{E}$ Y DEVRQ Y 789 WNVMDT F R 835 RLIHIF $R$ ORM D F $958 \underline{N}$ T_N_KEOR EYCSR

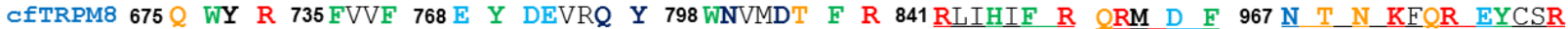
rTRPM8 $675 Q$ WY R 735 FVVF 768 E Y DEVRQ Y 798 WNVMDT F R 842 RLIHIF $R$ ORM D F $968 \underline{N}$ T_N_KEOR EYCNR hTRPM8 675 Q WY R 735 FVVF $768 \mathrm{E}$ Y DEVRQ Y 798 WNVMDT F R 842 RLIHIF $R$ ORM D F $968 \underline{\underline{N}}$ T_N_KEOR EYCSR hTRPM7 740 Q WM M 853IVKF $886 \mathrm{E}$ I EKVRE W 924 FNISDT F R 975 RLLDEL Q GKM N F 1085 Y V S KYOR AYHEK hTRPM6 $726 Q$ WM M 839 IVKF $872 \mathrm{E}$ S EVVRE W 910 WNLTET F R 952RLLDFF Q AKM N F $1058 \underline{Y}$ V S KYNR TYHEK hTRPM5 630 WW A 732VTVF 768 E Y EEIRQ Y 807 WNKCDM F R 848RLIHIF K ERM D F $967 \underline{N}$ T A KEOR EYHER

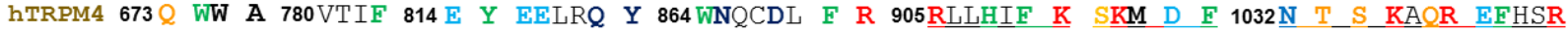
hTRPM3 $779 Q$ WM M 895 IVKF $928 \mathrm{E}$ I EKMRE W 967 WNVTDL F R 1008RLLDIF K GKM D M $1126 \underline{\underline{N}}$ T_S_KFOR TFHER hTRPM2 $736 Q$ WW V 796VVVF $829 \mathrm{E}$ Y EEMRQ Y 868 WNKLDV F R 909 RLMHIF K KRM D F 1037N T_T KEOR EYHGR hTRPM1 729 Q WM M 828 IVKF 861 E I EKIRE W 899 WNITDL F R 941RVLDIF K GKM D L 1064 N T_S KFOR TFHDR $\begin{array}{lllllllllllllllll}737 & 743 & 831 & 861865 & 875 & 878894 & 900 & 903 & 910917 & 941 & 945949 & 961 & 965967 & 1086 & 1091 & 10941101 & 1104\end{array}$

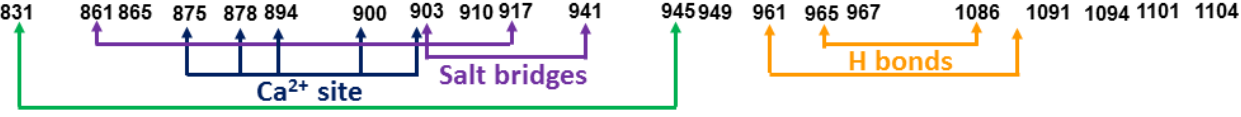

$\pi-\pi$ interaction

Figure 5 